\title{
Assessment of temporomandibular joint diseases in patients with rheumatoid arthritis by panoramic radiography and magnetic resonance imaging
}

\begin{abstract}
Objective: To assess the temporomandibular joint in patients with rheumatoid arthritis by both panoramic radiography and MRI.

Study design: Panoramic radiograph and MRI imaging were performed to forty joints of twenty patients from both sexes and varying ages, complaining of signs and/or symptoms of TMJ involvement. Diagnoses of Rheumatoid Arthritis were according to 1987 ACR criteria for RA. All images were analyzed and scored.

Results: Comparison between panorama and MRI revealed that there was statistically significant difference regard condyles position in panorama and condylar osseous changes in MRI, temporal cortical bone integrity in panorama and osseous condylar changes in MRIs and finally joints space narrowing in panorama and disc position, disc morphology, and joint effusion in MRIs. While, there was no statistically significant difference regard condyles morphology in panorama and osseous condylar changes in MRIs, and finally joint space narrowing in panorama and revealed retrodiscal band hyper intense signal detected in MR images.

Conclusion: There is a significant relation between TMJ involvement and RA disease called "TMD-RA". Early diagnosis and management of RA presents an important opportunity to alter the course of this progressive disease. MRI has a great role in detection of early involvement of TMJ in RA patients. This study recommends investigating TMJ in all diagnosed RA patients by MRI as a routine diagnostic tool due to its marvelous benefits.
\end{abstract}

Volume 5 Issue 4 - 2016

\author{
Tarek Abdallah Abd Elslame,' Maha Eshak, ${ }^{2}$ \\ Faten Esmail Mohame ${ }^{3}$ \\ 'Department of Oral and Dental Medicine, Minia General \\ Hospital, Egypt \\ ${ }^{2}$ Department of Oral Radiology Department, Faculty of \\ Dentistry, Minia University, Egypt \\ ${ }^{3}$ Department of Rheumatology and Rehabilitation Department, \\ Minia University, Egypt
}

\begin{abstract}
Correspondence: Maha Eshak, Department of Oral Radiology Department, Faculty of Dentistry, Minia University, Egypt,
\end{abstract} Emailmahaeshaq2@yahoo.com

Received: November 18, 2015 | Published: November 21, 2016

\section{Abbreviations}

RF, rheumatoid factor; RA, rheumatoid arthritis; ESR, erythrocyte sedimentation rate; CRP, C- reactive protein; TMD, temporo mandibular disease

\section{Introduction}

The rationale of the study is to find the relation between TMJ involvement and RA disease (TMD/RA). This study found that TMD signs and symptoms vary widely in RA patients ranging from, patients themselves, ignoring all TMD symptoms to sever pain, mandibular deviation during maximum extrusion, tinnitus, headache, and limited mandibular movements. However many patients don't report any problems concerning TMJ, they have sever TMJ involvement. ${ }^{1}$ TMJ is fibrous-cartilaginous joint surfaces separated by articular disk with two-sided simultaneous articulation; the unique biochemical composition of these articular tissues is reflected by material properties that are also distinct from those of other load-bearing articulations. ${ }^{2-4}$ Rheumatoid arthritis (RA) is a systemic disorder, which may involve many of the diarthroridal joints in the body. TMJ afflicted with RA may produce pain, joint stiffness, difficulties in opening the mouth, and open bite. The magnitude of TMJ involvement seems to be correlated with the severity of RA. The level of rheumatoid factor (RF), erythrocyte sedimentation rate (ESR), C-reactive protein (CRP), thrombocytes count, and plasma tumor necrosis factor- $\alpha$ have all been noted to correlate with the severity of TMD. ${ }^{5-13}$ Dental panoramic tomography has become a very popular radiographic technique in dentistry. The main reasons for this include: the technique is reasonably simple and the radiation dose is relatively low, particularly with rare-earth intensifying screens, the dose is equivalent to about three to four periapical radiographs. There was a good association between radiographic findings and subjective symptoms, and between radiographic findings and restricted condylar movement. However, to obtain a more detailed anatomic picture, Magnetic Reasons imaging (MRI) is suitable for patients with acute unexplained pain or as part of preoperative work-up. Genetic factors seem to contribute to destructive lesions in the TMJ as well as composition of saliva in patients with various rheumatic diseases. ${ }^{14}$ MRI is used to assess soft tissues, bone marrow changes, disc position, morphology, mobility, and joint effusion. It also plays an important role in the evaluation of temporomandibular disease (TMD), as it allows for a noninvasive depiction of the joint that is not otherwise available. The diagnostic accuracy of the clinical examination with (MRI) imaging is variable, ranging from $54 \%$ to $90 \%$.

\section{Patients and methods}

\section{Patient selection and inclusion criteria}

Twenty patients of both sexes and varying ages, selected randomly from the outpatient clinic of rheumatology department, Minia University hospital according to 1987 ACR criteria for RA and all of them had Signs and/or symptoms of TMJ involvement (TMJ sounds, crypts, pain, joint stiffness, and inability to open mouth or open bite). After approval of the research proposal by research ethics committee of faculty of dentistry, Minia university All cases were asked to sign a written consent explaining the nature and procedures of the study protocol. Only those who agree to sign the consent were included in the study. 


\section{Physical examination}

Include; General examination, musculoskeletal examination by Riche articular index, thorough intra-oral examination, and TMJ clinical examination: include a standardized clinical examination following the clinical assessment protocol for TMJ by Okeson. ${ }^{15}$

Laboratory tests: Routine investigations including CBC, ESR, Renal and liver functions were recorded.

Radiographic examination: Hand and Wrist radiographic examination: Experienced rheumatologists evaluated the joints most frequently involved in the RA. Conventional Radiographic examination (Panorama/TMJ protocol): Panoramic images were performed at the oral Radiology Department. Faculty of Dentistry, Minia University with "Orthoclase panoramic machine" (Trophy, France). Patients were instructed to avoid forceful swallowing or moving during sessions of machine rotations for Right and Left TMJs captures in the closed and open mouth positions on a single radiograph. Criteria of bony change in panoramic radiographs were recorded according to author, Helenius ${ }^{16}$ Magnetic Resonance Imaging: All the patients also fulfill the MRI questionnaire for TMJ and they were scanned with 1.5 Tesla superconductive magnet (Philips) using a head coil with the following imaging parameters: number of slices $=14$, slice thickness $=3 \mathrm{~mm}$, interslice gap $=1 \mathrm{~mm}$, $\mathrm{FOV}=150 * 1.2, \mathrm{Echo}=1 / 1$, Flip angle $=20, \mathrm{TR}=443$ and $\mathrm{TE}=23$. Three scout images were obtained; axial, coronal and sagittal with two pulse sequences in the para sagittal plane were taken while the patient was occluding in centric relation and with placing a 7 pieces of wooden tongue depressors in the patient's mouth with its long axis perpendicular to the mid-sagittal plane. T1, T2 weighted and Proton density spin echo images were obtained. The following measurement points were used for MR images. They comprise 13 linear, 2 angular and 8 ratio variables. They have been routinely used for MR images by many other authors as; ${ }^{17-19}$ Also Criteria of bony changes in MR images were recorded according to. ${ }^{20}$ Grading system developed as follows; ${ }^{21}$ Soft tissue changes score was divided into categories as

i. Disc Position: (0) for normal, If an anteriorly displaced disc with reduction and If anteriorly displaced disc without reduction.

ii. Disc Morphology: (0) If normal and If deformed.

iii. Joint effusion: (0) if absent, and (1) if present.

iv. Retrodiscal tissue: (0) if normal, and or hyper intense.
I. Bony changes score was divided into categories as follow

a. Condylar osseous changes

b. For, Normal condyle and joint structure

c. For, Mild irregularity in condylar destruction, bone marrow changes and minimal joint space narrowing.

d. For, Moderate or significant erosion in the condyle with joint space narrowing

e. For, Severe or complete bone destruction with obvious joint space narrowing.

f. Articular eminence / fossa changes scores According to presence or absence of erosion and deformation were categorized into: (0) if normal and (1) if deformed/changed.

\section{Results}

This study was conducted on 40 temporomandibular joints belonging to 20 individuals of both sexes; 3 males (15\%) and 17 females $(85 \%)$ whom are affected by rheumatoid arthritis disease with ages ranging from (31-74) years with mean age (49.95). Out of the 20 cases, 17 cases $(85 \%)$ had the onset of disease suddenly, $(50 \%)$ had a severe pain, and (35\%) had a limited mandibular movement. There were joint noise/sounds in $(85 \%)$, while $(45 \%)$ recalled joint pain during palpation, and (55\%) had signs of attrition/wear facets. Radiographic findings of hand \& wrist radiographs revealed that; $85 \%$ of cases had evidence of bone erosion distributed as $50 \%$ had large bone erosions in the involved surface area and 35\% had discrete erosions. $65 \%$ of all cases showed narrowed joint space distributed as $40 \%$ with focal narrowing, $20 \%$ narrowed joint space with reduction of less than $50 \%$, and one case had a reduction greater than $50 \%$ of joint space. Regarding conventional radiographs of 40 TMJs; $32.5 \%$ of examined joints had displaced condyle position, $65 \%$ showed changed condyle morphology Figure 1, 15\% showed affected integrity of articular temporal eminence and glenoid fossa, and $47.5 \%$ of joints showed reduced joint space area. From MR imaging, $45 \%$ of joints had disc displacement with reduction (Figure 2), 20\% had disc displacement without reduction, $45 \%$ had changed disc morphology, $67.5 \%$ of joints showed retrodiscal tissue band changed signal intensity, and $25 \%$ of all joints showed joint effusion in MR images (Table 1-3).

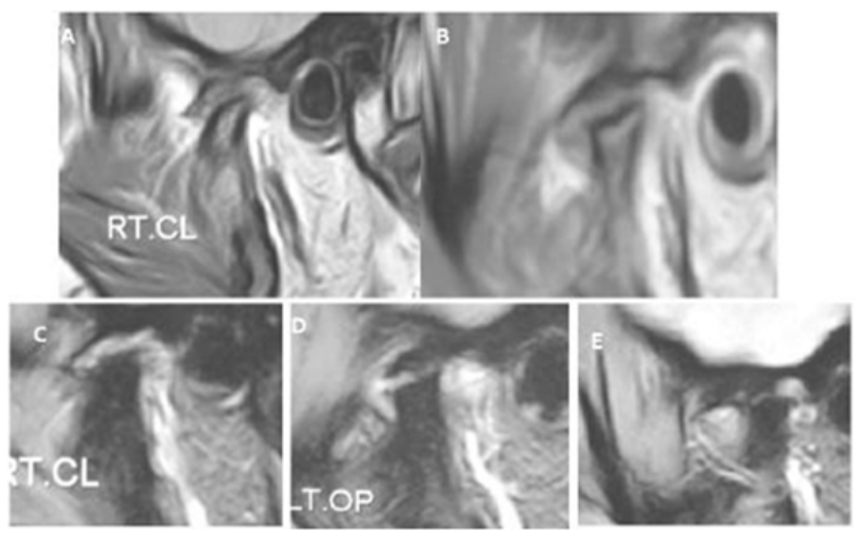

Figure I A female patient of 4 I years old with rheumatoid arthritis, suffering from open bite, noisy TMJ, clicking, tenderness during palpation and inability to open her mouth widely. (A) Sagittal proton density weighted image (closed-mouth position). (B) Sagittal proton density weighted image (closed mouth position) (C) SagittalT2weighted image with fat saturation (closed-mouth position). (D) Sagittal T2 weighted image with fat saturation (open mouth position). (A,C) For right TMJ (B, D, E) for left TMJ. Images showed severe condylar destructive changes, inhomogeneous signal intensity and joint effusion in both right and left joints. 

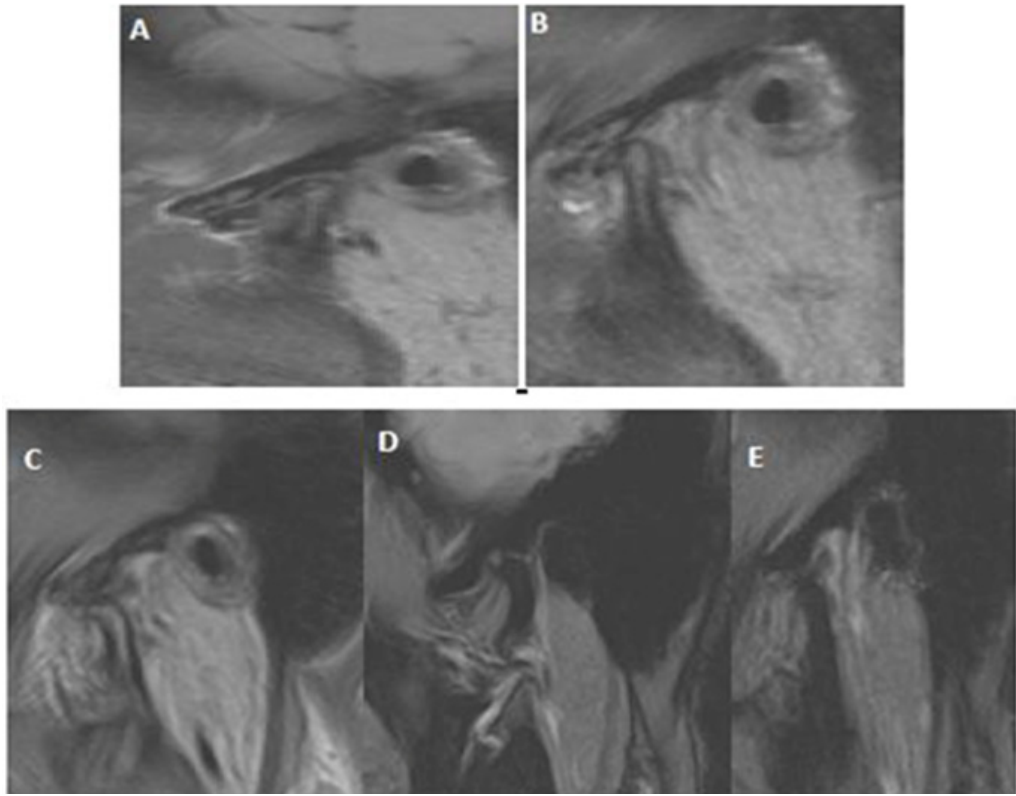

Figure $2 \mathrm{~A}$ female patient of 63 years old h RA from several years and on corticosteroid therapy from a long period. She is complaining from swelling and tenderness in her hand joints. Regarding TMJ, she recalls repeated dislocation in her jaw with protruded mandible and open bite. (A, B, C) Sagittal proton density weighted image (closed \&opened mouth position respectively) of the right and left TMJs. (D, E) T2 weighted images (closed \& open mouth position respectively). Images revealed condylar destructive and joint effusion and disc displacement with reduction.

Table I Relation between condyle position in (panorama) \& osseous condylar changes in MRI

\begin{tabular}{llllll}
\hline \multirow{2}{*}{ Variables } & & \multicolumn{2}{c}{ Condyle Position (Pan) } & \multirow{2}{*}{ R-Value } & P-Value \\
\cline { 3 - 5 } & & Normal & Displaced & & \\
\hline \multirow{2}{*}{ Right Condylar changes (MRI) } & Absent & $12(60 \%)$ & I (5\%) & \multirow{2}{*}{$5.93 *$} & 0.015 \\
& Present & $3(15 \%)$ & $4(20 \%)$ & & \\
\multirow{2}{*}{ Left Condylar changes (MRI) } & Absent & $6(30 \%)$ & $3(15 \%)$ & \multirow{2}{*}{0.3} & 0.582 \\
& Present & $6(30 \%)$ & $5(25 \%)$ & & \\
\hline
\end{tabular}

Table 2 Relation between cortical bone integrity of tubercle and fossa in (Panorama) \& condylar osseous changes in MRI

\begin{tabular}{llllll}
\hline \multirow{2}{*}{ Variables } & & \multicolumn{2}{c}{ Cortical Bone (Pan) } & \multirow{2}{*}{ R-Value } & \multirow{2}{*}{ P-Value } \\
\cline { 3 - 4 } & & Normal & Affected & & \\
\hline \multirow{2}{*}{ Right Condylar changes (MRI) } & Absent & $13(65 \%)$ & $0 \%$ & $4.12 *$ & 0.042 \\
& Present & $5(25 \%)$ & $2(10 \%)$ & & \\
\multirow{2}{*}{ Left Condylar changes (MRI) } & Absent & $9(45 \%)$ & $0 \%$ & & \multirow{2}{*}{0.043} \\
& Present & $7(35 \%)$ & $4(20 \%)$ & $4.09 *$ & \\
\hline
\end{tabular}

Table 3 Relation between Joint space narrowing in (panorama) \& retrodiscal tissue in MRI

\begin{tabular}{|c|c|c|c|c|c|c|}
\hline \multirow{2}{*}{\multicolumn{3}{|c|}{ Variables }} & \multirow{2}{*}{\multicolumn{2}{|c|}{ Joint Space Narrowing (Pan) }} & \multirow{2}{*}{ R-value } & \multirow{2}{*}{ P-Value } \\
\hline & & & & & & \\
\hline \multirow{2}{*}{ Right } & \multirow{2}{*}{ Retro-Disc (MRI) } & Normal & $\begin{array}{l}\text { Normal } \\
4(20 \%)\end{array}$ & $\begin{array}{l}\text { Reduced } \\
4(20 \%)\end{array}$ & \multirow{2}{*}{0.13} & \multirow{2}{*}{$0.7 \mid 4$} \\
\hline & & Hyper intense & 7 (35\%) & $5(25 \%)$ & & \\
\hline \multirow{2}{*}{ Left } & \multirow{2}{*}{ Retro-Disc (MRI) } & Normal & $3(15 \%)$ & $2(10 \%)$ & \multirow{2}{*}{0.26} & \multirow{2}{*}{0.606} \\
\hline & & Hyper intense & $7(35 \%)$ & $8(40 \%)$ & & \\
\hline
\end{tabular}

*Significant at $\mathrm{P} \leq 0.05$ 


\section{Discussion}

The diagnosis of RA is usually determined on the basis of a comprehensive evaluation of images, clinical findings and data on haematology, blood chemistry and urinalysis. This study has followed the diagnostic criteria established in 1987 by the American College of Rheumatology (ACR) in cases selection that are widely used in medical institutions all over the world. However, it has been reported that these criteria tend to be less sensitive in the early diagnosis of RA $^{22-25}$ TMJs affected by RA may produce pain, joint stiffness, difficulties in opening the mouth and open bite. RA shortens the life span by 5-7 years and if severe, significantly alters the normal life style. It is known that rheumatoid arthritis is a disease characterized by symmetrical joint involvement, explaining that $60 \%$ of our selected patients in this study had symmetrical TMJ involvement, ${ }^{26,27}$ The most prevalent TMD sign or symptom is joint sounds, as in this study it is obvious in $(85 \%)$ of examined joints, $(45 \%)$ recalled joint pain during palpation, while one case only $(0.5 \%)$ had a deviated mandible during maximum extrusion. Although some authors found that pain during condylar translation, asymmetry during maximum opening and protrusions as well as crepitation during movement were predictors for TMJ involvement with a good specificity but at low sensitivity. While other authors stated that "the clinical indicators of TMJ arthritis were reduced maximum mouth opening capacity, vertical differences between the two mandibular angular regions and deviation of the mandible". In contrast to reported studies stated that; subjective symptoms and abnormalities on physical examination were unreliable predictors of TMJ arthritis as detected by MRI, ${ }^{28,29} \mathrm{As} 20 \%$ of selected RA cases were asymptomatic but still had signs of TMDs, several explanations discussed why TMJ remains asymptomatic in RA patients although severe joint involvement by; subjective TMJ discomfort or complaints are likely to be overshadowed by joint problems elsewhere in the body like the hands and knees where frequent motion or weight-bearing is unavoidable in daily life, attaching more importance to other peripheral joint involvements that affect daily life activities and work lives bearing in mind the education and socio-economic levels of the region where the study was conducted. Also, due to the fact that is women are responsible for childcare and housekeeping chores. While regarding TMJ problems they can subjectively reduce its motion by talking less or by avoiding ingestion of hard food. Taking in consider that $85 \%$ of the selected patients in this study were females. ${ }^{27}$ This study was conducted to a high number of patients who have a positive radiographic finding in the hand \&wrist radiographs as bone erosion in $85 \%$ distributed as $50 \%$ with large bone erosions and $35 \%$ with discrete erosions. Also different grades of reduced joint space narrowing were assessed as $(40 \%)$ with focal narrowing, (20\%) with reduction of less than $50 \%$ of joint space and only (5\%) had reduction greater than $50 \%$ of joint space. TMJ lateral views showed that $80 \%$ had changed bone morphology in condyles, articular eminence, and temporal fossa while $47.5 \%$ had narrowed joint space. In consequence, this agrees with other authorswho found that the severity of radiologic changes in the TMJ was comparable to those of the MCP and MTP joints of the hands. However some stated that severe destruction of the TMJ due to RA, significantly more often in patients with severe cervical arthritis than in those without when evaluated by cervical radiography. ${ }^{10,30,31}$ $85 \%$ of the examined joints in this study by conventional TMJ radiography examining the integrity of the temporal eminence and fossa were found intact and condylar bony changes were recorded in $65 \%$ including destructive morphological changes as flattening, erosion of the mandibular condyle. This study goes in accordance with studies reported that the same typical radiological findings in RA patients regarding condylar assessment, and also to the other studies stated that osseous changes of the mandibular condyle are specific for RA cases. ${ }^{32}$

\section{Conclusion}

There is a significant relation between TMJ involvement and RA disease "TMD-RA". TMJ is affected in RA patients as any other synovial joint but it may appear later than small joints of hands, feet, and stress/weight bearing joints. Early diagnosis and management of RA presents an important opportunity to alter the course of this progressive disease. MRI has a great role in detection of early involvement of TMJ in RA patients compared to silent clinical complaints about TMJ. MRI of the TMJ results in no radiation load and facilitates representation of the articular hard-tissue and soft-tissue situation. MRI is accepted as the "gold standard" in imaging procedures for evaluating the TMJ in RA patients. So it is recommended to investigate TMJ in all diagnosed RA patients by MRI as a routine diagnostic tool due to its marvelous benefits. MRI findings are condylar deformation, osteophytes formation and erosions of the condylar compact or of the fossa articularis, degenerative changes in the condylar spongiosa, and intra-articular liquid accumulations in the bilaminar zone or joint space. Attention should be paid by dentists as many treatment planes could affect TMJ in RA as orthodontic treatments (bone erosions, deformities), prosthesis and full mouth rehabilitation (due to lost vertical dimension) and to oral and maxillofacial surgeries as RA may negatively contribute in results of many operations like fractures, Implantology and reconstruction operations as some RA patients is going on a long standing corticosteroid therapy; bone-resorption factor and RA itself enhance bone resorption.

\section{Acknowledgments}

None.

\section{Conflicts of interest}

The authors declare that there are no conflicts of interest.

\section{Funding details}

None.

\section{References}

1. Choi HK, Hernn MA, Seeger JD, et al. Methotrexate and mortality in patients with rheumatoid arthritis. Lancet. 2002;359(9313):1173-1177.

2. Fautrez J. Leiddraad bij de Studie van de Stelselmatige Ontleedkunde van de mens. Luik, Belgium, Europe: Uitgevers Desoer; 1967.

3. Alves N. Study about the Development of the Temporomandibular Joint in the Human Fetuses. Int J Morphol. 2008;26(2):309-312.

4. Badel T, Savić-Pavičin I, Zadravec D, et al. Temporomandibular joint development and functional disorders related to clinical otologic symptomatology. Acta Clin Croat. 2011;50(1):51-60.

5. Chalmers IM, Blair GS. Rheumatoid arthritis of the temporomandibular joint. J Med. 1973;166:369-386.

6. Akerman S, Kopp S, Nilner M, et al. Relationship between clinical and radiologic findings of the temporomandibular joint in rheumatoid arthritis. Oral Surgery Oral Medicine and Oral Pathol. 1988;66(6):639-643. 
7. Goupille P, Fouquet B, Goga D, et al. The temporomandibular joint in rheumatoid arthritis:correlations between clinical and tomographic features. J Dent. 1993;21(3):141-146.

8. Ash MM, Ramfjord SP. Disorders of occlusion and dysfunction of the masticatory system. In: Ash MM \& Ramfjord SP, editors, Occlusion, Pennsylvania, USA: WB Saunders; 1994. p. 164-194.

9. Çeliker R, Gokce-Kutsal Y, Eryilmaz M. Temporomandibular joint involvement in rheumatoid arthritis. Scand $J$ Rheumatology. 1995;24(1):22-25.

10. Yoshida A, Higuchi Y, Kondo M, et al. Range of motion of the temporomandibular joint in rheumatoid arthritis:relationship to the severity of disease. Cranio. 1998;16(3):162-167.

11. Nordahl S, Alstergren P, Eliasson S, et al. Radiographic signs of bone destruction in the arthritic temporomandibular joint with special reference to markers of disease activity:a longitudinal study. Rheumatology. 2001;40(6):691-694.

12. Liu HT, Chiu FY, Chen CM, et al. The combination of systemic antibiotics and antibiotics impregnated cement in primary total knee arthroplasty in patients of rheumatoid arthritis-evaluation of 60 knees. J Chin Med Assoc. 2003;66(9):533-536.

13. Voog U, Alstergren P, Eliasson S, et al. Inflammatory mediators and radiographic changes in temporomandibular joints of patients with rheumatoid arthritis. Acta Odontol Scand. 2003;61(1):57-64.

14. Larheim TA, Johannessen S, Tveito L. Abnormalities of the temporomandibular joint in adults with rheumatic disease:a comparison of panoramic, transcranial and transpharyngeal radiography with tomography. Dentomaxillofac Radiol. 1988;17(2):109-113.

15. Okeson JP. Management of temporomandibular disorders and acclusion. 5th edn. Mosby, St. Louis, Missouri, USA, 2003. p. 204.

16. Helenius LMJ. Oral and Temporomandibular joint findings in Rheumatic Diseases. Helsinki: Helsinki University; 2005.

17. Ozawa S, Boering G, Kawata $\mathrm{T}$, et al. Reconsideration of the $\mathrm{TM}$ condylar position during internal derangement: comparison between condylar position on tomogram and degree of disk displacement on MRI Cranio. 1999;17(2):93-100.

18. Pullinger AG, Seligman DA. Multifactorial analysis of differences in temporomandibular joint hard tissue anatomic relationships between disk displacement with and without reduction in women. J Prosthet Dent. 2001;86(4):407-419.

19. Gedrange T, Gredes T, Hietschold V, Dominiak M, et al. Comparison of reference points in different methods of temporomandibular joint imaging. Advances in Medical Sciences. 2012;57(1):157-162.
20. Wang EY, Fleisher KA. MRI of temporomandibular joint disorders. Appl radiol. 2008;37(9):17-25.

21. Abolmaali ND, Schmitt J, Schwarz W, et al. Visualization of the articular disk of the temporomandibular joint in near-real-time MRI:feasibility study. Eur Radiol. 2004;14(10):1889-1894.

22. Burket LW. Oral Medicine: Diagnosis and Treatment. USA: JB Lippincott Company; 1971. p. 7-23.

23. Pendleton N, Clague JE, Cobain M, et al. Relationship between self reported prevalence of diabetes mellitus using the Cornell Medical Index (CMI) and prevalence determined by glycosylated hemoglobin (HbA1c) in an elderly community- dwelling population. Arch Gerontol Geriatr. 2005;41(3):289-296.

24. Sakuragi S, Sugiyama Y. Effects of daily walking on subjective symptoms, mood abd autonomic Nervous Function. J Physiol Anthropol. 2006;25(4):281-289.

25. Kretapirom K, Okochi K, Nakamura S, et al. MRI characteristics of rheumatoid arthritis in the temporomandibular joint. Dentomaxillofac Radiol. 2013;42(4).

26. Lin Y, Hsu M, Yang J, et al. Temporomandibular joint disorders in patients with rheumatoid arthritis. J Clin Med Assoc. 2007;70(12):527-534.

27. Hiz O, Ediz L, Ozkan Y, et al. Clinical and Magnetic Resonance Imaging Findings of the Temporomandibular Joint in Patients with Rheumatoid Arthritis:Orginal Article. J Clin Med Res. 2012;4(5):323-331.

28. Twilt M, Schulten AJ, Nicolaas P, et al. Facioskeletal changes in children with Juvenile Idopathic arthritis. Ann Rheum Dis. 2006;65:823-825.

29. Pamela FW, Arabshahi B, Cahill AM, et al. High prevalence of temporomandibular joint arthritis at disease onset in chidren with juvenile idiopathic arthritis, as detected by magnetic resonance imaging but not by ultrasound. Arthritis Rheum. 2008;58(4):1189-1196.

30. Akerman S, Kopp S, Nilner M, et al. Relationship between clinical and radiologic findings of the temporomandibular joint in rheumatoid arthritis. Oral Surgery Oral Medicine and Oral Pathol. 1988;66(6):639-643.

31. Yamakawa M, Ansai T, Kasai S, et al. Dentition status and temporomandibular joint disorders in patients with rheumatoid arthritis. Cranio. 2002;20(30:165-171.

32. Harris ED, Budd RC, Firestein CS, et al. Kelley's Textbook of rheumatology. 7th ed. Elsevier Saunders. 2005;66:1043-1078.

33. Pendleton N, Clague JE, Horan MA, et al. Concordance of Cornell Medical Index self-reports to structured clinical assessment for the identification of physical health status. Arch Genrotol Geriatr. 2004;38(3):261-269. 